\title{
Probing interlayer interactions between graphene and metal substrates by supersonic rare-gas atom scattering
}

\author{
H. Shichibe, ${ }^{1}$ Y. Satake, ${ }^{1}$ K. Watanabe,${ }^{1}$ A. Kinjyo,${ }^{1}$ A. Kunihara, ${ }^{1}$ Y. Yamada,,${ }^{1, *}$ M. Sasaki, ${ }^{1}$ \\ W. W. Hayes, ${ }^{2}$ and J. R. Manson ${ }^{3,4}$ \\ ${ }^{1}$ Institute of Applied Physics, University of Tsukuba, 1-1-1 Tennodai, Tsukuba, Ibaraki, 305-8573, Japan \\ ${ }^{2}$ Physical Sciences Department, Greenville Technical College, Greenville, South Carolina 29606, USA \\ ${ }^{3}$ Department of Physics and Astronomy, Clemson University, Clemson, South Carolina 29634, USA \\ ${ }^{4}$ Donostia International Physics Center (DIPC), Paseo Manuel de Lardizabal, 4, 20018 Donostia-San Sebastian, Spain
}

(Received 18 December 2014; revised manuscript received 9 March 2015; published 6 April 2015)

\begin{abstract}
We demonstrate that highly surface-sensitive supersonic rare-gas ( $\mathrm{He}, \mathrm{Ar}$, and $\mathrm{Xe})$ atom scattering, in both the quantum and classical regimes, can probe and quantify the interlayer interactions between graphene monolayers and metal substrates in terms of the Debye temperature corresponding to the surface normal vibration, and the surface effective mass. As models of the strongly and weakly interacting graphene, we investigated two systems, graphene on $\mathrm{Ru}(0001)$ and $\mathrm{Pt}(111)$, respectively. The experimental data for $\mathrm{Ar}$ and $\mathrm{Xe}$ are compared with the results from theoretical simulations based on the classical smooth surface model. For $\mathrm{gr} / \mathrm{Pt}(111)$ we find that the scattering pattern of the rare-gas beam, including the Debye-Waller attenuation of the He beam, are quite similar to that from highly oriented pyrolytic graphite (HOPG); this suggests that the graphene-Pt(111) interaction is much like a van der Waals interaction. On the contrary, for the $\mathrm{gr} / \mathrm{Ru}(0001)$ system, we find a smaller Debye-Waller attenuation and a larger surface effective mass, indicating that graphene on $\mathrm{Ru}(0001)$ is tightly bonded to the substrate. Furthermore, asymmetrical spectral shapes in the Ar and Xe scattering spectra from $\mathrm{gr} / \mathrm{Ru}(0001)$ are interpreted as a result of the lateral distribution of the interlayer interaction corresponding to the moiré pattern. It is found that the "valley" region of the moiré pattern has high effective mass reflecting stronger bonding to the substrate, contributing to the high reflectivity of the He beam reported for this system. On the other hand, the effective mass of the "hill" region is found to be similar to that of HOPG, indicating that this region is well decoupled from the substrate. These results demonstrate a unique capability of atom scattering to probe and evaluate the molecule-substrate interaction and its spatial distributions.
\end{abstract}

DOI: 10.1103/PhysRevB.91.155403

PACS number(s): 61.05.Np, 63.22.Rc

\section{INTRODUCTION}

Interactions between organic semiconductors and metal electrodes are a central issue in organic electronics because these interactions determine carrier injection and transport in organic devices. It is important to understand the basic aspects of interlayer interactions using well-defined model systems. However, since the interaction between an organic molecule and the substrate is usually weak, conventional probes, such as $\mathrm{x}$-ray scattering and high-energy electron diffraction, become highly destructive. One of the few ideal probes for the investigation of fragile organic layers is supersonic rare-gas atomic beam scattering, in which the typical beam energy is in the range of ten to several hundred $\mathrm{meV}$ [1]. In particular, lowenergy elastic and inelastic He-atom scattering exhibits a high level of surface sensitivity and has been used for determining structural and vibrational properties of molecular adsorbates, which is not easy using other conventional techniques [2-4]. In this paper we use this sensitivity to obtain information about the weak interactions between substrates and a molecular overlayer through the detection of perpendicular vibrations of the molecular layer.

In the quantum mechanical scattering of a He-atom beam from an ordered molecular layer in which many atoms undergo elastic scattering (diffraction), vibrations of surface molecules reduce the coherence of scattered waves. The resulting thermal

\footnotetext{
*yamada@bk.tsukuba.ac.jp
}

attenuation of the diffraction intensity, known as Debye-Waller (D-W) attenuation, contains information about the vibration of the surface and can give the surface Debye temperature. For He-atom scattering at the specular diffraction peak, the $\mathrm{D}-\mathrm{W}$ attenuation can be written, in the standard approximation including a Beeby correction for the potential well depth [5], as

$$
I\left(T_{S}\right)=I_{0} \exp \left(-24 \frac{m}{M_{S}} \frac{\left(E_{\perp}+D\right)}{k_{B}} \frac{T_{S}}{\theta_{D n}{ }^{2}}\right) .
$$

Here $m$ and $M_{S}$ are the effective masses of the projectile and surface atom, respectively, $E_{\perp}$ is the energy of the incident beam corresponding to the motion in the surface normal direction, $D$ is the attractive potential well depth, $T_{S}$ is the surface temperature, and $\theta_{D n}$ is the surface Debye temperature. It is known that surface vibrational modes with large displacements in the surface normal direction, such as the Einstein mode of surface adsorbates, contribute significantly to diffuse scattering. Consequently, in He-atom scattering, the surface Debye temperature in Eq. (1) reflects the normal vibrational modes of the molecular layer [6]. Thus we denote the Debye temperature as $\theta_{D n}$ in this paper. Since vibrational modes polarized in the surface normal direction are largely determined by the force constant in molecule-substrate bonding, the $\theta_{D n}$ determined from (1) contains a fingerprint of molecule-substrate interactions.

In contrast, the specific nature of vibrations in the molecular layer becomes less important in classical inelastic scattering, which is readily realized in heavier rare-gas atomic 
beams, such as Ar, Kr, and Xe. Nevertheless, it is known that the surface normal vibration makes a large contribution to the classical scattering processes of rare-gas atoms. In this case, the peak position and spectral shape of the scattered atomic beam directly reflects information on the vibration of the overlayer. In this article we analyze inelastic scattering using a classical theory of heavier rare-gas scattering, namely the smooth surface model (SSM) [7-11]. The SSM describes scattering from a flat potential energy surface with vibrationally induced corrugation due to the vibrational motions of the surface atoms. This model has been used to successfully explain the scattering pattern and energy accommodation not only for flat surfaces but also for highly disordered liquid metals and metal alloys [11]. The differential reflection coefficient in the SSM is given by

$$
\begin{aligned}
& \frac{d R}{d \Omega_{f} d E_{f}} \\
& \propto \frac{\left|u_{f}\right|}{u_{i \perp}}\left|\tau_{f i}\right|^{2}\left(\frac{\pi}{k_{B} T_{S} \Delta E_{0}}\right)^{3 / 2} \\
& \quad \times \exp \left(-\frac{\left(E_{f}-E_{i}+\Delta E_{0}\right)^{2}+2 v_{R}^{2} m^{2}\left(u_{f_{/ /}}-u_{i_{/ /}}\right)^{2}}{4 k_{B} T_{S} \Delta E_{0}}\right),
\end{aligned}
$$

where $k_{\mathrm{B}}$ is the Boltzmann constant, $v_{R}$ is a weighted average of phonon velocities at the classical turning point of the beam $[12,13],\left|\tau_{f i}\right|^{2}$ is the scattering form factor, and $\Delta E_{0}$ is the recoil energy, which is given by $m^{2}\left(\boldsymbol{u}_{f}-\boldsymbol{u}_{\mathbf{i}}\right)^{2} / 2 M_{S}$.

In the present study the aforementioned sensitivity of elastic and inelastic rare-gas atom scattering to the surface-normal vibration of the overlayer is utilized to probe information about graphene-substrate interactions which is the well-defined model system. By measuring the surface Debye temperature and the surface effective mass of the graphenes, based on quantum mechanical and classical rare gas atom scattering, respectively, we are able to access and quantify the interlayer bonding, which would be quite difficult to probe using other conventional probes. This is a rather intuitive way to sense the "hardness" or "stiffness" of the graphene layers by "knocking on" them with inert projectiles. For this purpose we focus on single-layer graphene on different substrates as well-defined model systems having different interlayer interactions. We use single-layer graphene on $\mathrm{Ru}(0001)$ and $\mathrm{Pt}(111)$ [i.e., $\mathrm{gr} / \mathrm{Ru}(0001)$ and $\mathrm{gr} / \mathrm{Pt}(111)]$ as models of strongly and weakly interacting graphenes, respectively. The results of rare-gas scattering from these graphenes are compared to that from HOPG, which serves as a reference for nearly freestanding graphene supported by the van der Waals interaction. Graphene on $\operatorname{Pt}(111)$ is known as the model of nearly freestanding graphene [14-21]. Density functional theory (DFT) calculations have shown that the graphene-substrate distance of $\operatorname{gr} / \operatorname{Pt}(111)$ is approximately $3.3 \AA$, which is nearly the same as the $3.354 \AA$ interlayer distance in HOPG [14,18-20]. Core-level photoemission has also shown nearly unaltered $\mathrm{C} 1 s$ spectra [11], suggesting that the graphene is indeed nearly freestanding on the $\mathrm{Pt}(111)$ substrate. In contrast, $\mathrm{gr} / \mathrm{Ru}(0001)$ has a huge corrugation on the surface (a moiré corrugation), reflecting a lateral mismatch in the interlayer interaction [22-33].
It has been reported that the interlayer distance of the "hill" region of the moiré corrugation is 3.3-3.7 $\AA$ and that of the "valley" is around $2.2 \AA$ [24,26,28,34]. It has also been reported that the electronic state of graphene on $\mathrm{Ru}(0001)$ is greatly altered, which reflects stronger bonding to the substrate [33].

Although graphene overlayers on $\mathrm{Ru}(0001)$ and $\operatorname{Pt}(111)$ (and HOPG) should have similar "lateral" bonding due to strong C-C bonds, their "perpendicular" bonding to the substrate, to which the atom scattering is sensitive, differs depending on the substrates. We indeed find that rare-gas scattering from graphene on the two substrates clearly differs, reflecting the different natures of interlayer interactions. The scattering spectra from $\mathrm{gr} / \mathrm{Pt}(111)$ are similar to those from HOPG, suggesting the freestanding nature of graphene. However, gr/Ru(0001) exhibited significantly different features, such as a large surface Debye temperature and a large surface effective mass, indicating that the graphene on $\mathrm{Ru}(0001)$ exhibited a stiffer surface due to its strong bonding to the $\mathrm{Ru}(0001)$ substrate. We also found that Ar and Xe scattering spectra from $\mathrm{gr} / \mathrm{Ru}(0001)$ exhibit significant asymmetries in their angular distributions, possibly reflecting the lateral distribution of the interlayer interaction corresponding to the moiré corrugation. It is found that the valley region of the moiré pattern on $\mathrm{gr} / \mathrm{Ru}(0001)$ has a high effective mass reflecting stronger bonding of graphene to the substrate. This is attributable to the high reflectivity of He beam scattering reported for this system $[35,36]$. On the other hand, the effective mass of the hill region is found to be similar to that of HOPG, indicating that this region is well decoupled from the substrate and supported by van der Waals force. The present results suggest the "lateral resolution" of the atom scattering for the distribution of the force constant to be approximately $3 \mathrm{~nm}$, at least. It is thus demonstrated that atom scattering in both the quantum mechanical and classical regimes can probe and evaluate molecule-substrate interactions and their lateral distributions.

\section{EXPERIMENTAL DETAILS}

All the experiments were conducted with a supersonic molecular beam apparatus whose details are described elsewhere [37]. Supersonic atomic beams of He and Ar were generated by a jet nozzle with a $50-\mu \mathrm{m}$ diameter aperture. The incident translational energy of the rare-gas beam was controlled by adjusting the nozzle temperature and by mixing with a carrier gas. We used pure He gas without seeding for the He-atom scattering measurements; translational energies ranged from 11 to $67 \mathrm{meV}$, depending on the temperature of the nozzle. Ar and Xe were seeded with $\mathrm{He}$ at a mixture ratio of 1:9, resulting in translational energies of $340 \mathrm{meV}$ for $\mathrm{Ar}$ and $504 \mathrm{meV}$ for $\mathrm{Xe}$ at a nozzle temperature of $300 \mathrm{~K}$. He, Ar, and Xe scattering spectra were measured by rotating the sample with the angle between incident beam and detector fixed at $90^{\circ}$.

Single layers of graphene on $\operatorname{Pt}(111)$ and $\mathrm{Ru}(0001)$ were formed by thermal cracking of backfilled ethylene at the surface with temperatures of 1100 and $1300 \mathrm{~K}$, respectively. A clean HOPG surface was prepared by cleaving the crystal in air before transfer to an ultrahigh vacuum (UHV) chamber. The formation of graphene was examined by lowenergy electron diffraction (LEED) and scanning tunneling 


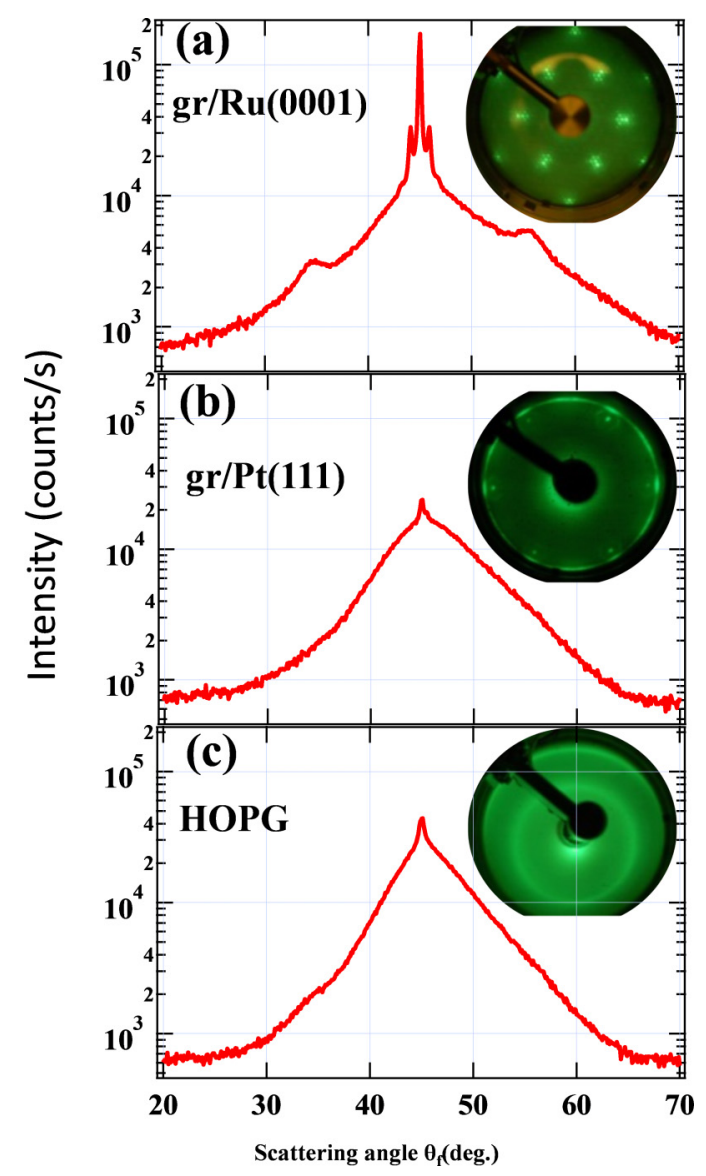

FIG. 1. (Color online) Angular distribution spectra as functions of final scattering angle $\theta_{f}$ for He scattering with incident beam energy of $67 \mathrm{meV}$ : (a) He scattering taken in the $\langle 112\rangle$ direction from a single layer of graphene on $\mathrm{Ru}(0001)$, (b) from single layer graphene on $\mathrm{Pt}(111)$, and (c) from clean HOPG. The inset in each panel shows the corresponding LEED pattern.

microscopy (STM). The sample temperature was measured with a thermocouple attached directly to the sample. Most scattering measurements (except the D-W attenuation measurements) were conducted using the sample at room temperature.

\section{RESULTS AND DISCUSSION}

\section{A. HAS of graphene layers}

The graphene layers were characterized by He diffraction and LEED measurements. Figures 1(a)-1(c) display the $\mathrm{He}$ diffraction spectra and the LEED images from $\mathrm{gr} / \mathrm{Ru}(0001)$, $\operatorname{gr} / \mathrm{Pt}(111)$, and HOPG, respectively. For all spectra, the incident energy of the He beam was set to $67 \mathrm{meV}$. The LEED images for graphene on $\mathrm{Ru}(0001)$ and $\mathrm{Pt}(111)$ agree with those in previous reports. The LEED image for $\mathrm{gr} / \mathrm{Ru}(0001)$ shows clear diffraction spots from the moiré pattern [23,30]. In the $\mathrm{He}$ diffraction spectrum, small but distinct diffraction peaks due to the moiré pattern appear at about half a degree on either side of the specular peak at $45^{\circ}$ (parallel $k$ vector of $\pm 0.25 \AA^{-1}$ ). A detailed $\mathrm{He}$ diffraction spectrum from the moire pattern with a low-energy He beam of $12.8 \mathrm{meV}$ is shown in Fig. 2(a); in
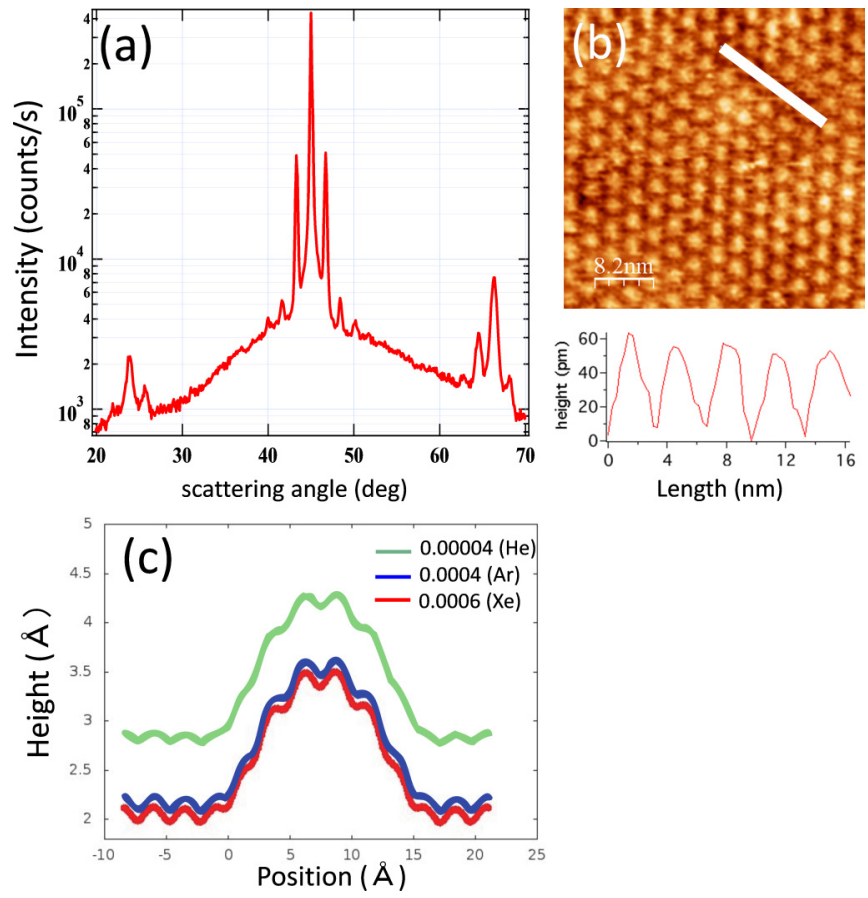

FIG. 2. (Color online) (a) He atom angular distribution spectra for an incident energy of $12.8 \mathrm{meV}$ taken in the $\langle 112\rangle$ direction, showing specular and first order diffraction graphite peaks and secondary peaks from the moiré corrugation of a single graphene layer on $\mathrm{Ru}(0001)$. (b) The upper panel shows an STM pattern of the moiré pattern and the lower panel exhibits a single line trace along the high symmetry direction. (c) Equicharge density surface of $\mathrm{gr} / \mathrm{Ru}(0001)$ at the classical turning points of $\mathrm{He}, \mathrm{Ar}$, and Xe, taken from Ref. [38].

addition to $1 \times 1$ diffraction peaks due to the graphene lattice, the figure exhibits several fractional-order diffraction peaks due to moiré periodicity. The presence of well-defined moiré corrugation also appears in the STM image of the same sample, as shown in Fig. 2(b). These observations verify the formation of a high-quality, single-layer graphene.

The equicharge density surfaces of the $\mathrm{gr} / \mathrm{Ru}(0001)$ at the turning point of the $\mathrm{He}, \mathrm{Ar}$, and Xe beams with conditions used in this study are shown in Fig. 2(c). The charge density plot was calculated by Campi et al., by means of density functional theory (DFT) calculations within the local density approximation (LDA) [38], The plot shows the line profile across the center of the moiré unit cell. The vertical axis shows the relative height of the classical turning points from the carbon atoms in the flat area (valley region) of the moiré corrugation. It is found that the corrugation of the charge density shows a bump of approximately $1.5 \AA$ for all the projectiles. It is also found that the shape of the charge-density surface does not depend significantly on the charge density relevant to this study ranging from 0.00004 to $0.0006 e / a_{0}^{3}$. It is noted that, in our fixed source-sample-detector geometry of the scattering measurements, the incident condition depends on the scattering angle. Therefore, projectiles are reflected from slightly different charge-density surface within one spectrum. However, this effect is considered to be negligible in the present study where the shape of the charge-density surface is almost independent of the actual value of the charge density. 
The LEED image of gr/Pt(111) shows a slightly segmented ring pattern in addition to the $1 \times 1$ spots of the $\mathrm{Pt}(111)$ lattice, suggesting the presence of rotated domains as a result of a weak interlayer interaction $[14,20,39,40]$. The He diffraction spectra of gr/Pt(111) and gr/Ru(0001) differ significantly. The specular reflection intensity of the He beam from $\mathrm{gr} / \mathrm{Ru}(0001)$ was much larger than those from gr/Pt(111) and HOPG. In addition, the inelastic background due to diffuse scattering of He was smaller for $\mathrm{gr} / \mathrm{Ru}(0001)$, while it was predominant in the He diffraction spectra of HOPG and gr/Pt(111). These clear differences in He diffraction spectra may initially appear to be somewhat surprising because the topmost layer of these systems, to which the He beam is sensitive, was graphene for all samples. However, these differences in the He diffraction spectra mainly reflect differences in interlayer interactions. From the above observations, it can be surmised that graphene on $\mathrm{Ru}(0001)$ is much "stiffer" and "harder" than the other graphenes. In contrast, He diffraction from gr/Pt(111) is very similar to that from HOPG. This similarity indicates that the interaction between graphene and $\operatorname{Pt}(111)$ is much more like a van der Waals interaction as it is in HOPG.

\section{B. Surface Debye temperatures}

To investigate the differences in interlayer-interactions of these graphene systems in a more quantitative way, we evaluated the surface Debye temperatures $\left(\theta_{D n}\right)$ corresponding to the surface-normal vibrations of graphene, based on D$\mathrm{W}$ attenuation measurements of the specular $\mathrm{He}$ diffraction intensity. Figure 3 shows the logarithmic D-W attenuation plots of the specular reflection of each He beam as functions of surface temperature for the three graphene systems. For all the measurements, the He incident energy was set to $67 \mathrm{meV}$. To determine the Debye temperature using Eq. (1), we used a "standard" value of the surface attractive potential well depth $D$ of $16 \mathrm{meV}$, according to previous studies [4,11,41]. Note

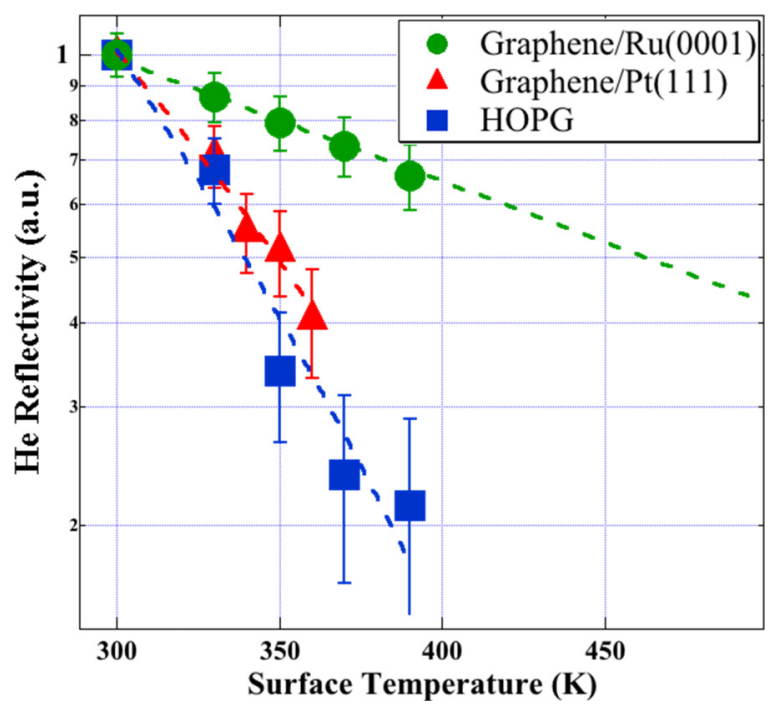

FIG. 3. (Color online) Debye-Waller plots as a function of surface temperature for the $\mathrm{He}$ specular peak at an incident energy of $67 \mathrm{meV}$. The triangle data points are for HOPG, squares for $\mathrm{gr} / \mathrm{Pt}(111)$, and circles for $\mathrm{gr} / \mathrm{Ru}(0001)$. that, in Fig. 3, the specular intensities are normalized at the values for room temperature. Figure 3 shows that the decay of the specular intensity from $\mathrm{gr} / \mathrm{Ru}(0001)$ clearly differs from that of HOPG and $\mathrm{gr} / \mathrm{Pt}(111)$, while $\mathrm{gr} / \mathrm{Pt}(111)$ shows similar D-W attenuation to that of HOPG. This may suggest that $\mathrm{gr} / \mathrm{Ru}(0001)$ has a larger $\theta_{D n}$ than the other graphenes, while those of gr/Pt(111) and HOPG are similar. Note that the Debye temperature considered here is one corresponding to the surface-normal vibration to which $\mathrm{He}$ is particularly sensitive, not the one corresponding to the $\mathrm{C}-\mathrm{C}$ bonds in the graphene which should be nearly constant for the three systems.

However, there is a well-known problem with such an assumption which is clear from Eq. (1) where it is seen that the squared Debye temperature appears multiplied by the surface effective mass. Thus an unambiguous determination of the Debye temperature can be made only if the crystal mass $M_{S}$ is known, which is not necessarily the case for composite or molecular surfaces such as those considered here. Nevertheless, if it is assumed that the mass $M_{S}$ is $12 \mathrm{amu}$, corresponding to the mass of a single carbon atom, the $\theta_{D n}$ values deduced from the slope of the Debye-Waller attenuation based on (1) are $1130 \pm 50,580 \pm 70$, and $480 \pm 70 \mathrm{~K}$ for $\mathrm{gr} / \mathrm{Ru}(0001)$, gr/Pt, and HOPG, respectively. These values of $\theta_{D n}$ agree with previous reports using the same technique $[4,36,41]$. Despite the ambiguity associated with determination of the crystal mass, it is clear that the $\theta_{D n}$ of $\operatorname{gr} / \operatorname{Pt}(111)$ is similar in value to the HOPG within the uncertainty, but that of $\mathrm{gr} / \mathrm{Ru}(0001)$ is significantly larger, approximately twice as large as that of the other two graphenes.

\section{Surface effective masses from Ar scattering}

The ambiguity in the effective crystal mass can be alleviated by carrying out measurements of atom-surface scattering in the classical regime. This is seen from Eq. (2) where the effective mass appears independently and there is no association with the Debye temperature. The Debye temperature does not appear in Eq. (2) because it is in the classical limit of large numbers of phonons excited in the collision, and because the number of phonons transferred is large, all quantum coherence is lost and even the explicit nature of the phonon density becomes unimportant. However, Eq. (2) can be derived starting from fundamental quantum principles and then taking the classical limit of large numbers of phonon quanta, in which case the derivation demonstrates that the crystal mass appearing in the Debye-Waller factor of Eq. (1) is identical to the mass appearing in the classical scattering transition rate of Eq. (2) $[12,13]$. Thus, the mass ambiguity problem associated with evaluations of the Debye temperature via thermal attenuation measurements of diffraction peaks can be lifted by determining the effective mass from independent measurements of scattering spectra taken under classical conditions in which all diffraction is suppressed. For this reason, we have performed a series of measurements of the in-plane scattered angular distributions of hyperthermal $\mathrm{Ar}$ and Xe scattering from the same three substrates as for the $\mathrm{He}$ atom beams in Figs. 1-3. These experimental conditions are clearly in the classical regime and allow for determinations of the effective crystal mass associated with Ar or Xe for each of the three different substrates. Of course what is needed 


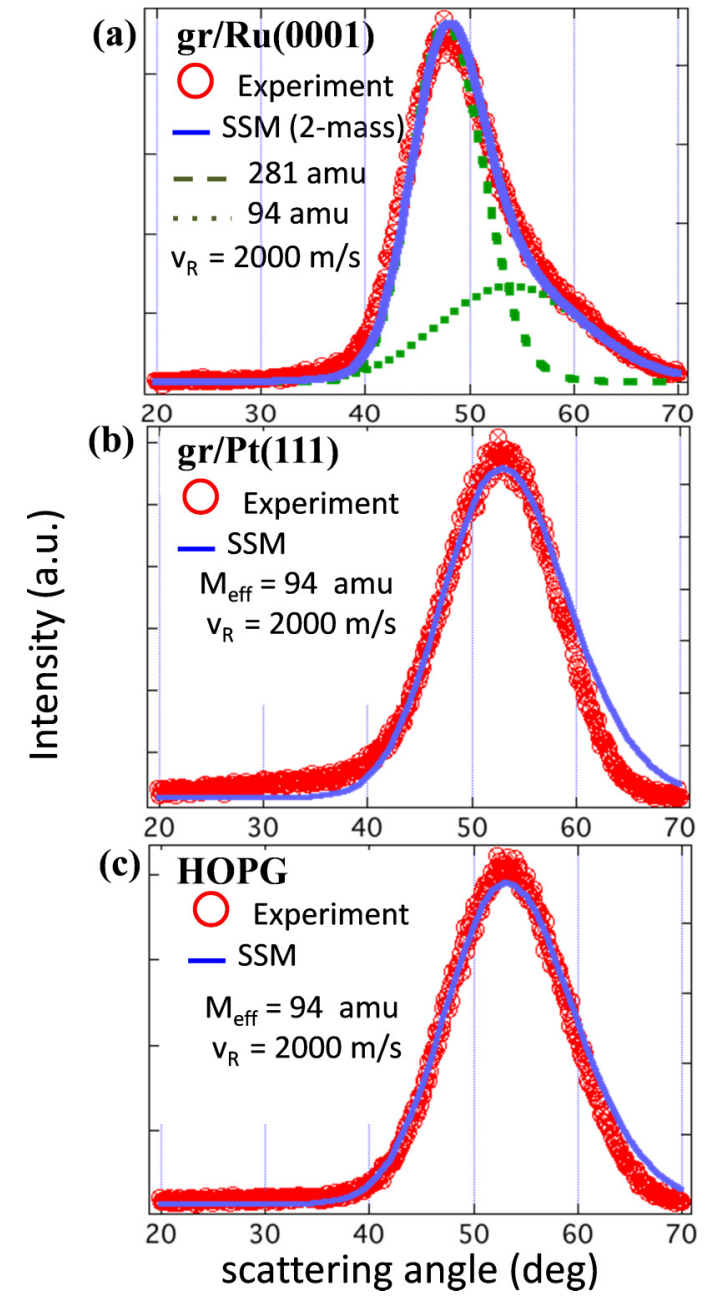

FIG. 4. (Color online) Angular distributions for Ar, with incident energy of $340 \mathrm{meV}$, scattering from three different surfaces, with experimental data shown as points and the smooth curves are the calculations as explained in the text: (a) single layer graphene on $\mathrm{Ru}(0001)$, (b) single layer graphene on $\mathrm{Pt}(111)$, and (c) graphite (HOPG).

is an independent measurement of the effective mass for He atom scattering, but this would require incident energies and/or surface temperatures much larger than is possible with the current experimental apparatus. However, the classical measurements with $\mathrm{Ar}$ and $\mathrm{Xe}$, in addition to determining the effective mass, provide further evidence of the distinctly different nature of the $\mathrm{gr} / \mathrm{Ru}(0001)$ system as compared with the somewhat similar nature of the graphene bonding to $\mathrm{Pt}(111)$ and HOPG.

Figure 4 shows Ar scattering spectra from $\mathrm{gr} / \mathrm{Ru}(0001)$, gr/Pt(111), and HOPG, which were obtained for an Ar beam with a translational energy of $340 \mathrm{meV}$. The figure shows a clear difference between the Ar scattering spectra from $\mathrm{gr} / \mathrm{Ru}(0001)$ and $\operatorname{gr} / \operatorname{Pt}(111)$, while the scattering spectrum from $\mathrm{gr} / \mathrm{Pt}(111)$ is similar to that of HOPG. Ar scattering from gr/Pt(111) and HOPG exhibits broad and symmetric peaks centered at the final scattering angle of approximately $53^{\circ}$. In contrast, $\mathrm{gr} / \mathrm{Ru}(0001)$ produces a rather sharp and intense peak at a scattering angle of approximately $47^{\circ}$, which is rather close to the specular position. This indicates that the projectile feels a much heavier effective mass for $\mathrm{gr} / \mathrm{Ru}(0001)$. To extract the effective masses for each sample, we utilized a simulation based on the smooth surface model of Eq. (2). In the fitting process we took the effective mass $M_{S}$ and $v_{\mathrm{R}}$ as variable parameters. The dashed curve in Fig. 4(a) and solid curves in Figs. 4(b) and 4(c) show results from the simulation using the SSM. The experimental spectra of gr/Pt(111) and HOPG are well reproduced by the SSM, but the fitting is significantly less good for the case of $\mathrm{gr} / \mathrm{Ru}(0001)$. From the best fit of each spectrum, we deduced the effective masses to be 281, 94, and $90 \mathrm{amu}$ for $\mathrm{gr} / \mathrm{Ru}(0001)$, gr/Pt(111), and HOPG, respectively. The calculations were not strongly sensitive to the value of $v_{\mathrm{R}}$ which was taken to be $2000 \mathrm{~m} / \mathrm{s}$ for all calculations shown, a value consistent with previous results for Ar and Xe scattering from molten metals [11]. Increasing or decreasing $v_{\mathrm{R}}$ by a factor of $50 \%$ changes the calculations almost imperceptibly.

It is immediately evident that the deduced surface effective masses are significantly larger than the single carbon mass, suggesting that a collective interaction takes place in the Ar-graphene collision. The effective mass of $90 \mathrm{amu}$ for HOPG suggests that the single Ar atom interacts with 7.5 carbon atoms, corresponding to the mass slightly larger than that of one benzene ring. The similar effective mass found for $\mathrm{gr} / \mathrm{Pt}(111)$ indicates that the topmost graphene layers in these systems are almost equivalent and that they are almost freestanding or supported by the van der Waals force as in the case of HOPG. On the other hand, the effective mass of $\mathrm{gr} / \mathrm{Ru}(0001)$ is considerably larger than the other graphenes. The mass 281 amu can be considered, for example, as equivalent to one benzene ring plus two $\mathrm{Ru}$ atoms underneath the graphene layer. This much larger effective mass of $\mathrm{gr} / \mathrm{Ru}(0001)$ can be considered to be a fingerprint for strong bonding of graphene to the substrate and is responsible for the apparent high value of $\theta_{D n}$ deduced from the $\mathrm{D}-\mathrm{W}$ attenuation of the diffracted He beam.

Previous studies of rare-gas and diatomic molecular scattering from clean $\mathrm{Ru}(0001)$ have revealed a large effective mass. An analysis of the energy-resolved spectra of $\mathrm{Ar}$ scattering from $\mathrm{Ru}(0001)[42,43]$ as well as scattering of $\mathrm{N}_{2}$ molecules from similar $\mathrm{Ru}(0001)$ [44,45] gave for both projectile particles an effective mass of approximately $2.5 \mathrm{Ru}$ atoms. Based on the analysis of those two systems it was proposed that the $\mathrm{Ru}(0001)$ surface would present a large effective mass for the scattering of all the rare gases and even for other projectiles [46]. The implication of this prediction, as seen from the Debye-Waller factor of Eq. (1), is that under quantum mechanical conditions the diffraction peaks for scattering of light rare gases such as $\mathrm{He}$ or $\mathrm{Ne}$ would have a much weaker than expected thermal attenuation, i.e., they would have a much larger than expected elastic reflectivity. Such a large elastic reflectivity has indeed been observed for He scattering from $\mathrm{Ru}(0001)$ [47] and also subsequently for He scattering from graphene covered $\mathrm{Ru}(0001)$ [36].

\section{Two-mass simulations using SSM}

As shown by the dashed curves in Fig. 4(a), the spectral shape of Ar scattering from $\mathrm{gr} / \mathrm{Ru}(0001)$ is not completely reproduced by the SSM. The experimental spectral shape 
exhibits noticeable asymmetry with respect to the peak maximum, the sort of asymmetry that can be fitted with two Gaussian-like peaks with one shifted in position and much smaller than the principle peak. Recognizing that the hexagonal hill-and-valley shape of the graphene moiré corrugation may well lead to stronger bonding in the valley regions and weaker bonding in the hill regions suggests that the effective mass may in fact vary across the moiré unit cell. In order to simulate such an effect we make the simple assumption that the moiré unit cell consists of a mixture of two regions with different effective masses. Under this assumption we have fitted the spectra with two components, the first being the SSM with the same effective mass and a second component with a smaller effective mass similar to that found for the case of scattering from HOPG, i.e., 90 amu. For Ar scattering from $\mathrm{gr} / \mathrm{Ru}(0001)$ the dotted curve shown in Fig. 4(a) shows the contribution of this second component normalized to 0.35 in intensity with respect to the mass 281 amu SSM component, and the solid curve is the combined intensity of the sum of the two components. The combined intensity matches the asymmetry in the data quite well, suggesting that the hill-like region of the graphene is nearly freestanding and exhibits an effective mass that is close to HOPG. In contrast, the valley-like region is tightly bonded to the substrate and exhibits increased effective mass. From the fitting process, the intensity ratio of the two components with effective masses of 281 and 90 was deduced to be $0.74: 0.26$. This ratio can be considered to be a measure of the area of the valleys and hills in the moire corrugation of graphene on $\mathrm{Ru}(0001)$. Indeed, the area ratio of the valleys and the hills estimated from the charge density surface of graphene/Ru(0001) at the turning point of Ar beam shown in Fig. 2(c) [38] reveals that the ratio of the valley and the hill to be approximately $0.77: 0.23$, which matches the ratio of the two components in the SSM analysis. Furthermore, a similar value has been reported for the branching ratio of $\mathrm{C} 1 \mathrm{~s}$ photoemission spectrum in this system $[17,33]$. The splitting of the $\mathrm{C} 1 s$ spectrum has also been interpreted as due to the presence of the two distinct regions, i.e., weakly and strongly interacting portions of graphene, in the moiré corrugation of the graphene.

Thus, our analysis of Ar scattering on $\mathrm{gr} / \mathrm{Ru}(0001)$ using two-mass SSM reveals that the topmost surface can be modeled as a mixture of two regions with different effective mass, and that these two regions are, most likely, the valley and hill regions of the moiré corrugation of the graphene. In addition, the two-mass analysis suggests that the effective mass of the hill region is similar to that of HOPG, while a very large effective mass is found for the valley region. We therefore find that the hill region of the graphene is well decoupled from the substrate and possibly supported by van der Waals forces. However, the valley region seems to be tightly bound to the substrate. This fact contributes to the high reflectivity of the He beam, making this system attractive as a possibility for a mirror of a He atom microscope $[35,36]$.

Here we briefly discuss the applicability of this technique to molecular layers in general. In the case of a monolayer of (small) molecules, He atom scattering sensitively detects the Einstein-like vibrational modes of the molecule, therefore probing the molecule-substrate bonding [4]. It is then important to determine the lateral resolution of the molecule-substrate bonding in this technique. Since the atomic projectiles interact with several surface atoms in one collision event, the intrinsic resolution is limited. Therefore the intramolecule resolution of the molecule-substrate bonding will be difficult to determine, although not impossible. On the other hand, the aforementioned sensitivity to the moire distribution of the graphene-substrate bonding indicates that lateral resolution is better than $3 \mathrm{~nm}$. Therefore, molecular-level resolution of the molecule-substrate interaction will eventually be achieved, and this can be utilized in the determination of the molecule-substrate bonding in inhomogeneous systems, such as amorphous layers or molecules on inhomogeneous substrates, that appear in more realistic systems.

\section{E. Xe scattering}

Similar results were also obtained for Xe scattering, as shown in Fig. 5. Figure 5(a) shows an angular distribution spectrum for $507 \mathrm{meV}$ Xe scattering from HOPG with a surface temperature of $300 \mathrm{~K}$. The SSM calculation, which is the solid line, fits the data quite well with an effective mass of $252 \mathrm{amu}$, which is equal to the mass of 3.5 graphene rings. Figure 5(b) shows similar angular distribution data for $\mathrm{Xe}$ scattering from graphene covered $\mathrm{Ru}(0001)$ at $507 \mathrm{meV}$. As pointed out previously for Ar scattering from $\mathrm{gr} / \mathrm{Ru}(0001)$, the single peak is narrower and nearer to the specular position than that for scattering from HOPG, and there is a very
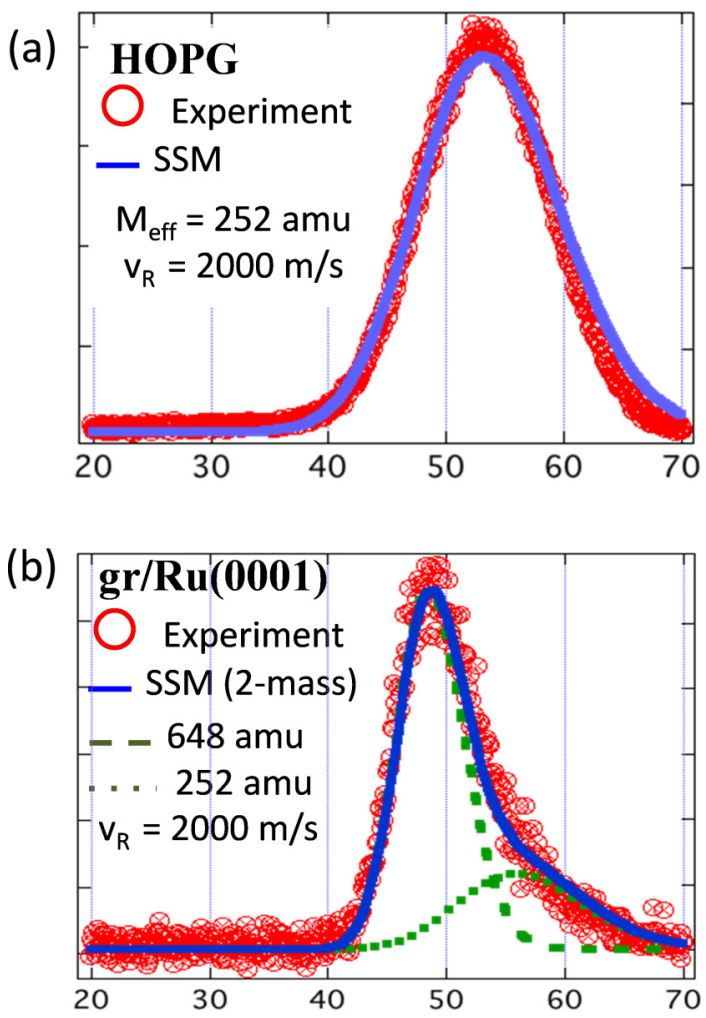

FIG. 5. (Color online) (a) Angular distribution for $507 \mathrm{meV} \mathrm{Xe}$ scattering from clean, ordered graphite (HOPG), experimental data shown as points and the calculation is the solid curve, and (b) similarly, angular distribution for $507 \mathrm{meV}$ Ar scattering from single layer of graphene on $\mathrm{Ru}(0001)$. 
marked asymmetry in the tail of the peak at supraspecular angles. The SSM calculation shown as a dashed curve, with an effective mass of 720 amu (approximately 10 graphene rings) qualitatively explains the narrower peak and its nearer to specular position. However, it does not explain the large-angle asymmetry evident in the experimental points. Using the two-mass model described for the case of Ar scattering, the entire spectrum is well explained as shown by the combined contributions in the solid line. In Fig. 5(b) the dotted curve shows an SSM contribution equal in intensity to 0.15 of the principal contribution in the dashed curve, and this secondary contribution is calculated with the HOPG effective mass of $252 \mathrm{amu}$. The solid curve is the sum of both the principle contribution with an effective mass of $720 \mathrm{amu}$ and the secondary contribution with effective mass of $252 \mathrm{amu}$. Note that the latter effective mass is the same as that of HOPG, again indicating the decoupled nature of the hill region. The valley-hill ratio deduced from the Xe scattering is 0.83:0.17, which differs slightly from the results from the Ar scattering. This difference may be due to the difference in the potential energy surface at the turning points of these projectiles. However, from Fig. 2(c) it is found that the shape of the potential energy surface at the turning point of Xe does not differ so much from that for Ar. Therefore, the difference is more likely to be due to the small signal to noise ratio in the Xe experiment.

\section{F. Possible effects from rainbow scattering}

The above discussion reveals that $\mathrm{Ar}$ and Xe scattering from $\mathrm{gr} / \mathrm{Ru}(0001)$ is well reproduced using the SSM under the assumption that the in-plane distribution of the surface effective mass due to the moiré pattern can be represented by a combination of two different masses. However, the asymmetry in the Ar and Xe scattering from gr/Ru(0001) might also be due to the geometric corrugation corresponding to the moiré pattern corrugation, namely, due to classical rainbow scattering. We simulated the effect of classical rainbow scattering using the washboard model of Tully (WBM) [48] assuming a sinusoidal corrugation approximating the electronic density surface. For the surface corrugation, we used an amplitude of $0.15 \mathrm{~nm}$ and a period of $3 \mathrm{~nm}$, based on the calculated charge density surface shown in Fig. 2(c). The results of the simulations of Ar scattering spectra from $\mathrm{gr} / \mathrm{Ru}(0001)$ using the WBM are displayed in Fig. 6 together with the experimental spectrum. The figure shows that the overall fitting is not particularly good, and all spectra simulated by the WBM exhibit much wider peak widths than the experimental spectrum, even though we simulated various surface effective masses. In particular, classical rainbow scattering produces a strong peak at scattering angles smaller than specular position, which is absent from the experimental data. Thus, the simple sinusoidal surface corrugation model does not seem to provide a particularly good explanation of Ar and Xe scattering from the moiré corrugation.

\section{SUMMARY}

We have demonstrated that, because of high sensitivity to vertically polarized vibrations of the graphene layer, quantum

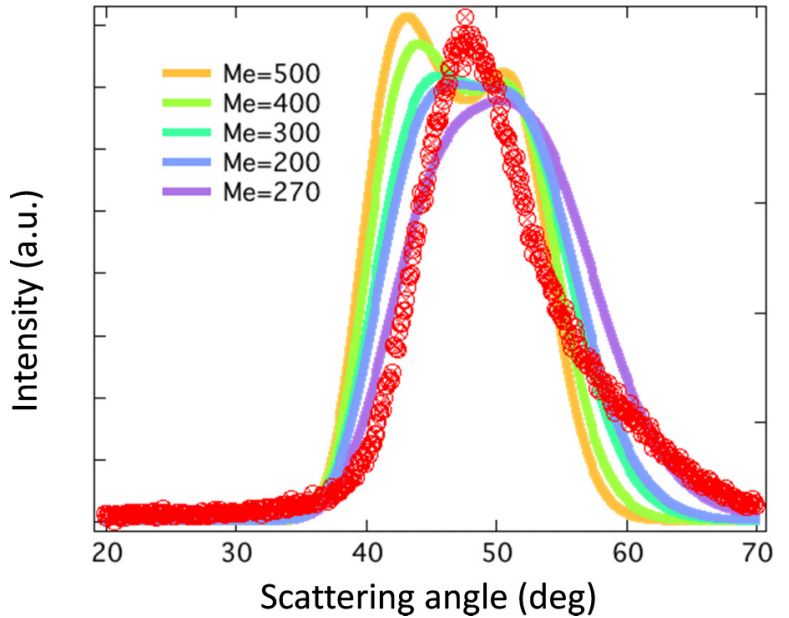

FIG. 6. (Color online) Comparison of washboard model calculations with the experimental data of Fig. 4 for Ar scattering from a graphene layer on $\mathrm{Ru}(0001)$. The smooth curves are calculations for different effective masses as explained in the text.

mechanical and classical rare-gas atom scattering can be useful tools for probing graphene-substrate interactions. Using $\mathrm{He}$ scattering, we found that $\mathrm{D}-\mathrm{W}$ attenuation of the diffraction intensity by $\mathrm{gr} / \mathrm{Pt}(111)$ was similar to that of HOPG, but it was much weaker for $\mathrm{gr} / \mathrm{Ru}(0001)$. These findings appear to reflect the differences in the graphene-substrate interactions, which characterize the vertical vibrations. The deduced surface Debye temperature of $\operatorname{gr} / \operatorname{Pt}(111)(580 \pm 70 \mathrm{~K})$ was similar to that of HOPG $(480 \pm 70 \mathrm{~K})$, but that of $\mathrm{gr} / \mathrm{Ru}(0001)$ is much larger $(1130 \pm 50 \mathrm{~K})$, suggesting that the interlayer interaction of $\mathrm{gr} / \mathrm{Pt}(111)$ was van der Waals-like while that in $\mathrm{gr} / \mathrm{Ru}(0001)$ was much stronger.

From the results of inelastic Ar scattering, we deduced that the surface effective mass of $\mathrm{gr} / \mathrm{Pt}(111)$ (94 amu) was similar to that of HOPG (90 amu), while that of $\mathrm{gr} / \mathrm{Ru}(0001)$ (281 amu) was approximately three times larger than that of HOPG. This also confirms that graphene is almost freestanding on $\mathrm{Pt}(111)$, while it is strongly bonded to $\mathrm{Ru}(0001)$. Furthermore, $\mathrm{Ar}$ and $\mathrm{Xe}$ scattering from $\mathrm{gr} / \mathrm{Ru}(0001)$ could be described as an in-plane distribution of the effective mass corresponding to the moiré pattern, revealing the presence of an in-plane distribution of the graphene-substrate interaction corresponding to the moiré corrugation. We found that the hill region of graphene was decoupled from the substrate, while the valley region was tightly bound to the substrate; this leads to a heavier effective mass and contributes to high reflectivity for a He beam. These findings indicate strong potential of atom scattering to probe and evaluate the interlayer bonding and its lateral distributions.

\section{ACKNOWLEDGMENTS}

We would like to thank Professor G. Benedek and D. Campi for fruitful discussions and for providing us with the charge density plot of $\mathrm{gr} / \mathrm{Ru}(0001)$. This work was supported by JSPS KAKENHI Grants No. 23360019, No. 24760023, and No. 26286011. 
[1] D. Farias and K.-H. Rieder, Rep. Prog. Phys. 61, 1575 (1998).

[2] G. Benedek, M. Bernasconi, V. Chis, E. Chulkov, P. M. Echenique, B. Hellsing, and J. Peter Toennies, J. Phys.: Condens. Matter 22, 084020 (2010).

[3] Y. Yamada, Phys. Rev. Lett. 99, 196105 (2007).

[4] Y. Yamada, C. Sugawara, Y. Satake, Y. Yokoyama, R. Okada, T. Nakayama, M. Sasaki, T. Kondo, J. Oh, J. Nakamura, and W. W. Hayes, J. Phys.: Condens. Matter 22, 304010 (2010).

[5] J. L. Beeby, J. Phys. C: Solid State Phys. 4, L359 (1971).

[6] G. Comsa, K. Kern, and B. Poelsema, Helium Atom Scatering from Surfaces (Springer, Berlin, 1992).

[7] J. R. Manson, Phys. Rev. B 43, 6924 (1991).

[8] J. R. Manson, Comput. Phys. Commun. 80, 145 (1994).

[9] W. W. Hayes and J. R. Manson, Phys. Rev. B 89, 045406 (2014).

[10] W. W. Hayes and J. R. Manson, Phys. Rev. Lett. 109, 063203 (2012).

[11] W. W. Hayes and J. R. Manson, J. Chem. Phys. 127, 164714 (2007).

[12] R. Brako and D. M. Newns, Phys. Rev. Lett. 48, 1859 (1982).

[13] R. Brako, Surf. Sci. 123, 439 (1982).

[14] H. Zi-Pu, D. F. Ogletree, M. A. Van Hove, and G. A. Somorjai, Surf. Sci. 180, 433 (1987).

[15] T. A. Land, T. Michely, R. J. Behm, J. C. Hemminger, and G. Comsa, Surf. Sci. 264, 261 (1992).

[16] T. A. Land, T. Michely, R. J. Behm, J. C. Hemminger, and G. Comsa, J. Chem. Phys. 97, 6774 (1992).

[17] A. B. Preobrajenski, M. L. Ng, A. S. Vinogradov, and N. Mårtensson, Phys. Rev. B 78, 073401 (2008).

[18] P. Sutter, J. T. Sadowski, and E. Sutter, Phys. Rev. B 80, 245411 (2009).

[19] M. Gao, Y. Pan, C. Zhang, H. Hu, R. Yang, H. Lu, J. Cai, S. Du, F. Liu, and H.-J. Gao, Appl. Phys. Lett. 96, 053109 (2010).

[20] M. Gao, Y. Pan, L. Huang, H. Hu, L. Z. Zhang, H. M. Guo, S. X. Du, and H.-J. Gao, Appl. Phys. Lett. 98, 033101 (2011).

[21] G. W. Cushing, V. Johánek, J. K. Navin, and I. Harrison, J. Phys. Chem. C 119, 4759 (2015).

[22] A. L. Vázquez de Parga, F. Calleja, B. Borca, M. C. G. Passeggi, J. J. Hinarejos, F. Guinea, and R. Miranda, Phys. Rev. Lett. 100, 056807 (2008).

[23] D. Martoccia, P. R. Willmott, T. Brugger, M. Björck, S. Günther, C. M. Schlepütz, A. Cervellino, S. A. Pauli, B. D. Patterson, S. Marchini, J. Wintterlin, W. Moritz, and T. Greber, Phys. Rev. Lett. 101, 126102 (2008).

[24] B. Wang, M.-L. Bocquet, S. Marchini, S. Günther, and J. Wintterlin, Phys. Chem. Chem. Phys. 10, 3530 (2008).

[25] D. Jiang, M.-H. Du, and S. Dai, J. Chem. Phys. 130, 074705 (2009).

[26] W. Moritz, B. Wang, M.-L. Bocquet, T. Brugger, T. Greber, J. Wintterlin, and S. Günther, Phys. Rev. Lett. 104, 136102 (2010).

[27] B. Borca, S. Barja, M. Garnica, M. Minniti, A. Politano, J. M. Rodriguez-García, J. J. Hinarejos, D. Farías, A. L. V. de Parga, and R. Miranda, New J. Phys. 12, 093018 (2010).
[28] D. Stradi, S. Barja, C. Díaz, M. Garnica, B. Borca, J. J. Hinarejos, D. Sánchez-Portal, M. Alcamí, A. Arnau, A. L. Vázquez de Parga, R. Miranda, and F. Martín, Phys. Rev. Lett. 106, 186102 (2011).

[29] D. Stradi, S. Barja, C. Díaz, M. Garnica, B. Borca, J. J. Hinarejos, D. Sánchez-Portal, M. Alcamí, A. Arnau, A. L. Vázquez de Parga, R. Miranda, and F. Martín, Phys. Rev. B 85, 121404 (2012).

[30] K. Katsiev, Y. Losovyj, Z. Zhou, E. Vescovo, L. Liu, P. A. Dowben, and D. W. Goodman, Phys. Rev. B 85, 195405 (2012).

[31] M. Iannuzzi, I. Kalichava, H. Ma, S. J. Leake, H. Zhou, G. Li, Y. Zhang, O. Bunk, H. Gao, J. Hutter, P. R. Willmott, and T. Greber, Phys. Rev. B 88, 125433 (2013).

[32] R. Cortés, D. P. Acharya, C. V. Ciobanu, E. Sutter, and P. Sutter, J. Phys. Chem. C 117, 20675 (2013).

[33] D. Alfè, M. Pozzo, E. Miniussi, S. Günther, P. Lacovig, S. Lizzit, R. Larciprete, B. S. Burgos, T. O. Menteş, A. Locatelli, and A. Baraldi, Sci. Rep. 3, 2430 (2013).

[34] D. Martoccia, M. Björck, C. M. Schlepütz, T. Brugger, S. A Pauli, B. D. Patterson, T. Greber, and P. R. Willmott, New J. Phys. 12, 043028 (2010).

[35] P. Sutter, M. Minniti, P. Albrecht, D. Farías, R. Miranda, and E. Sutter, Appl. Phys. Lett. 99, 211907 (2011).

[36] A. Politano, B. Borca, M. Minniti, J. J. Hinarejos, A. L. Vázquez de Parga, D. Farías, and R. Miranda, Phys. Rev. B 84, 035450 (2011).

[37] T. Kondo, D. Mori, R. Okada, M. Sasaki, and S. Yamamoto, J. Chem. Phys. 123, 114712 (2005).

[38] D. Campi and G. Benedek (unpublished).

[39] M. Sasaki, Y. Yamada, Y. Ogiwara, S. Yagyu, and S. Yamamoto, Phys. Rev. B 61, 15653 (2000).

[40] Y. Yamada, A. Sinsarp, M. Sasaki, and S. Yamamoto, Jpn. J. Appl. Phys. 41, 7501 (2002).

[41] J. P. Oh, T. Kondo, D. Hatake, and J. Nakamura, Surf. Sci. 603, 895 (2009).

[42] B. Berenbak, S. Zboray, B. Riedmuller, D. C. Papageorgopoulos, S. Stolte, and A. W. Kleyn, Phys. Chem. Chem. Phys. 4, 68 (2002).

[43] W. W. Hayes and J. R. Manson, Phys. Rev. B 75, 113408 (2007).

[44] H. Mortensen, E. Jensen, L. Diekhöner, A. Baurichter, A. C. Luntz, and V. V. Petrunin, J. Chem. Phys. 118, 11200 (2003).

[45] H. Ambaye and J. R. Manson, J. Chem. Phys. 125, 176101 (2006).

[46] W. W. Hayes, H. Ambaye, and J. R. Manson, J. Phys.: Condens. Matter 19, 305007 (2007).

[47] M. Minniti, C. Díaz, J. L. F. Cuñado, A. Politano, D. Maccariello, F. Martín, D. Farías, and R. Miranda, J. Phys.: Condens. Matter 24, 354002 (2012).

[48] J. C. Tully, J. Chem. Phys. 92, 680 (1990). 